\title{
Dermoide ocular em bovino - Relato de caso
}

\author{
Ocular dermoid in cattle - Case report \\ Dermoid de ojos en ganado - Reporte de caso
}

Recebido: 20/04/2021 | Revisado: 10/04/2021 | Aceito: 14/04/2021 | Publicado: 27/04/2021

\author{
Murilo Resende Silva \\ ORCID: https://orcid.org/0000-0002-9950-123X \\ Centro Universitário de Mineiros- UNIFIMES, Brasil \\ E-mail: murilo.resende@ hotmail.com \\ Lara Giovana Diniz \\ ORCID: https://orcid.org/0000-0002-4254-1062 \\ Centro Universitário de Mineiros- UNIFIMES, Brasil \\ E-mail: laradiniz@unifiems.edu.br \\ Nathalie Moro Bassil Dower \\ ORCID: https://orcid.org/0000-0003-3564-5258 \\ Universidade Federal de Mato Grosso, Brasil \\ E-mail: nathaliedower@gmail.com \\ Lianna Ghisi Gomes \\ ORCID: https://orcid.org/0000-0003-0909-2426 \\ Universidade Federal de Mato Grosso, Brasil \\ E-mail: liannaghisi@gmail.com \\ Juliana Evangelista Bezerril \\ ORCID: https://orcid.org/0000-0002-6352-4341 \\ Centro Universitário de Mineiros- UNIFIMES, Brasil \\ E-mail: julianaevb@unifimes.edu.br \\ Fabrício Eumar de Souza \\ ORCID: https://orcid.org/0000-0002-5566-7705 \\ Centro Universitário de Mineiros- UNIFIMES, Brasil \\ E-mail: fabricio@unifimes.edu.br \\ Priscila Chediek Dall'Acqua \\ ORCID: https://orcid.org/0000-0001-5781-4492 \\ Centro Universitário de Mineiros- UNIFIMES, Brasil \\ E-mail: priscila.chediek@unifimes.edu.br \\ Andresa de Cássia Martini \\ ORCID: https://orcid.org/0000-0002-2553-3685 \\ Centro Universitário de Mineiros- UNIFIMES, Brasil \\ E-mail: andresa.martini@unifimes.edu.br
}

\begin{abstract}
Resumo
O objetivo do estudo foi relatar um caso de dermoide ocular unilateral na terceira pálpebra de um bovino com três semanas de idade, abordando os aspectos diagnósticos, bem como a importância da conduta terapêutica para manutenção zootécnica e qualidade de vida do animal. A confirmação diagnóstica foi realizada por meio de exame histopatológico, sendo a exérese da massa realizada mediante a elevação da terceira pálpebra e incisão em cunha na região da lesão, obtendo êxito cirúrgico e sem recidivas. Concluiu-se que o cisto dermoide mesmo sendo pouco prevalente nos rebanhos bovinos, não deve ser negligenciado, pois as complicações oftálmicas geradas pela sua permanência em estruturas oculares podem ser graves. O estudo enfatizou a importância do diagnóstico e conduta terapêutica precoces, para manutenção da qualidade de vida do animal e manutenção de ganhos zootécnicos em animais de produção.
\end{abstract}

Palavras-chave: Anexos cutâneos; Cisto; Olho; Pele.

\begin{abstract}
The dermoid is a cystic mass of normal skin-like consistency located in an anatomically abnormal region, containing cutaneous attachments such as hair, stratified squamous epithelium, hair follicles, sebaceous glands, and other structures. The aims of this article is to report a case of unilateral third eyelid dermoid in a 3-week-old bovine, addressing the diagnostic aspects, as well as the importance of therapeutic conduct for animal maintenance and quality of life. Diagnostic confirmation was carried out through histopathological analysis, with the excision of the mass performed with elevation of the third eyelid and wedge incision in the region of the lesion, obtaining surgical success and without recurrences. It is concluded that the dermoid cyst, even though it is of low occurrence in bovine herds, should not be neglected, since there are great ophthalmic complications caused by its permanence in the eyeball. This
\end{abstract}


study emphasizes the importance of early diagnosis and therapeutic conduct, for maintaining the animal's quality of life and maintaining zootechnical gains for farm animals.

Keywords: Cutaneous attachments; Cyst; Eye; Skin.

\section{Resumen}

El dermoide es una masa quística de consistencia cutánea normal ubicada en una región anatómicamente anormal, que contiene inserciones cutáneas como cabello, epitelio escamoso estratificado, folículos pilosos, glándulas sebáceas y otras estructuras. El objetivo de este artículo es reportar un caso de dermoide unilateral del tercer párpado en un bovino de 3 semanas, abordando los aspectos diagnósticos, así como la importancia de la conducta terapéutica para el mantenimiento y la calidad de vida del animal. La confirmación diagnóstica se realizó mediante análisis histopatológico, realizándose la exéresis de la masa con elevación del tercer párpado e incisión en cuña en la región de la lesión, obteniendo éxito quirúrgico y sin recidivas. Se concluye que el quiste dermoide, a pesar de que es de baja ocurrencia en el ganado bovino, no debe descuidarse, ya que existen grandes complicaciones oftálmicas provocadas por su permanencia en el globo ocular. Este estudio enfatiza la importancia del diagnóstico temprano y la conducta terapéutica, para mantener la calidad de vida del animal y mantener las ganancias zootécnicas para los animales de granja.

Palabras clave: Inserciones cutáneas; Quiste; Ojo; Piel.

\section{Introdução}

As afecções oftálmicas são cada vez mais frequentes na clínica de grandes animais, apresentando riscos significativos para a capacidade de visão, o que pode causar depreciação do valor zootécnico do animal. O dermoide ocular é uma afecção pouco comum e com baixa incidência em bovinos, mas com significativa importância na clínica veterinária (Brudenall, Ward, Kerr, \& Newman, 2008; Roh, Gi, Lim, \& Kim, 2014; Almeida et al., 2018).

O dermoide pode se desenvolver em várias partes do corpo, como globo ocular e anexos, narinas (Brudenall et al., 2008), cavidade bucal (Souza, Nogueira, Turatti, Menezes Júnior, \& Freitas, 2008), sistema reprodutor (Souza, Pereira, Andrade, Moura, Mileo, Lima, \& Jaques., 2020) e sistema nervoso (Canelas, Cruz, \& Tenuto, 1960). Em casos raros, o cisto pode se desenvolver em regiões ósseas (Steinmetz et al., 2009). O dermoide foi descrito em várias espécies como bovinos, búfalos, equinos, camelos, ovinos, cães, gatos, coelhos e no ser humano (Wagner, Brügmann, Drommer, \& Fehr, 2000; Sarrafzadeh-Rezaei, Farshid, \& Saifzadeh, 2007; Brudenall et al., 2008; Albuquerque, Freitas, Hünning, Bercht, \& Pigatto, 2012).

O dermoide caracteriza-se pelo crescimento de tecido normal de consistência firme semelhante a pele em uma região anatomicamente anormal, podendo se manifestar em várias estruturas do globo ocular como as pálpebras, terceira pálpebra, córneas, conjuntiva e outras estruturas perioculares (Barkyoumb \& Leipold, 1984; Almeida et al., 2018).

Sua origem ainda não encontra- se totalmente elucidada, mas presume-se que seu surgimento se inicie a partir de uma massa congênita benigna de origem embrionária, com derivações de camadas ectodérmicas, mesodérmicas e mais raramente células de origem endodérmica (Muñoz, Leiva, Naranjo, \& Pena, 2007; Stelmann, Souza, Araujo, Monteiro, Seppa, \& Silva, 2009; Dantas et al., 2010; Carniel \& Leseux, 2018).

Em bovinos, o dermoide ocorre esporadicamente nos anexos oculares, sem predileção de gênero e se apresenta mais comumente na forma unilateral ou com menor frequência na forma bilateral (Barkyoumb \& Leipold, 1989; Brudenall et al., 2008).

Em alguns casos, o dermoide ocular se apresenta apenas como um cisto de coloração esbranquiçada que não atinge com gravidade a visão do animal, causando apenas irritação, e que pode ser facilmente removido pela ceratectomia lamelar superficial (Cunha, 2008; Carniel \& Leseux, 2018).

Contudo, algumas complicações podem ocorrer dependendo do tamanho da estrutura e do local onde se apresenta (Brudenall et al., 2008; Cunha, 2008). De acordo com Barkyoumb e Leipold (1989), as estruturas que mais manifestaram o dermoide ocular foram as pálpebras, terceira pálpebra e córnea, sendo que o tratamento de eleição é a exérese cirúrgica (Parrah 
et al., 2013).

É de grande importância a realização de um diagnóstico preciso e precoce da enfermidade, possibilitando descartar doenças semelhantes e consequentemente evitar danos a visão do animal, destacando-se que é fácil a identificação desta anomalia. Contudo, sua detecção pode ocorrer de modo tardio dependendo de sua natureza e extensão (Leipold, 1984).

O dermoide pode se apresentar de modos variados e aspectos e com isso se assemelha a outras patologias, assim é importante o diagnóstico diferencial, o qual pode auxiliar no tratamento e avaliação quanto a origem da enfermidade (Albuquerque et al., 2012). O diagnóstico diferencial pode ser realizado por meio de exame histopatológico, possibilitando a diferenciação e identificação das células e estruturas presentes, e associando estes achados com as características macroscópicas da massa (Stelmann et al., 2009).

Utumi, Zambon, Pedron, Machado e Rocha (2010) e Albuquerque et al. (2012) descreveram que o diagnostico diferencial deve distinguí-lo do cisto epidermóide. Contudo também deve ser realizado para descartar neoplasias na região ocular, lesões cicatriciais, cistos de inclusão epitelial, infecções, massas granulosas e lipoma (Stelmann et al., 2009).

$\mathrm{O}$ artigo relata um caso de dermoide ocular unilateral na terceira pálpebra de um bovino com três semanas de idade, abordandoas pectos diagnósticos, bem como a importância da conduta terapêutico-cirúrgica para manutenção zootécnica e qualidade de vida do animal.

\section{Relato de Caso}

Foi atendido na Fazenda Experimental Luís Eduardo de Oliveira Salles (FELEOS) no Município de Mineiros, Goiás, Brasil, um bezerro mestiço do cruzamento da raça Nelore com Gir leiteiro com três semanas de idade, pesando aproximadamente $30 \mathrm{~kg}$, o qual segundo proprietário, não estava conseguindo ingerir o leite, apresentando refluxo nasal. Ao exame físico, o animal apresentava-se em estação, mucosas de coloração rosa claro, temperatura corpórea de $39{ }^{\circ} \mathrm{C}$, frequência cardíaca de 102 batimentos/min. e frequência respiratória de 30 movimentos/min. Ao exame da cavidade oral observou-se defeito em fenda palatina que se estendia da porção rostral do palato duro até a porção distal do palato mole. Ao exame oftálmico, observou-se massa anormal de coloração amarelo esbranquiçada de consistência firme na terceira pálpebra direita, com tamanho aproximado de $0,5 \mathrm{~cm}$ e presença de pelos em sua superfície (Figura 1A).

Figura 1A - Presença de massa de consistência firme, de cor amarelo esbranquiçada, com presença de pelos na superfície da terceira pálpebra direita de bovino com 3 semanas. 1B- Fotomicrografia de dermoide (HE, objetiva de 4x). É possível observar epitélio escamoso estratificado (seta preta), folículos pilosos (seta vermelha) e glândulas sebáceas (ponta de seta preta).

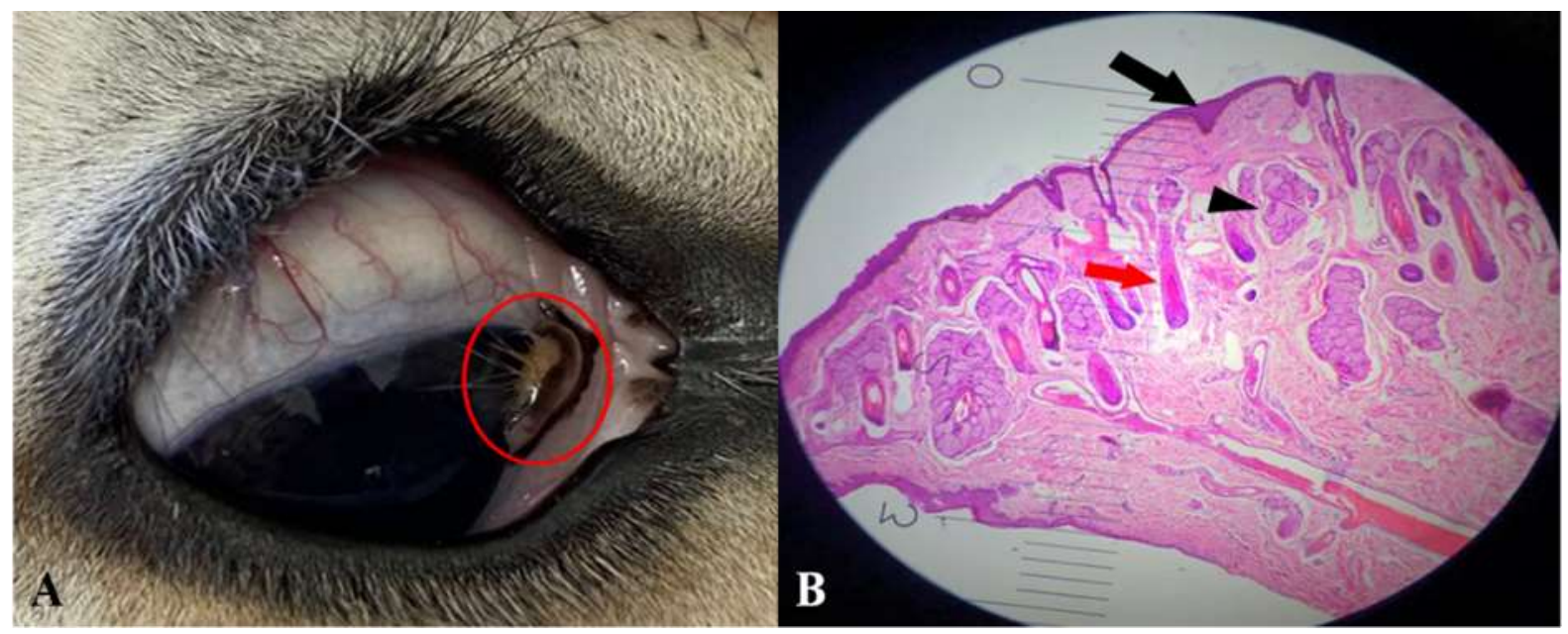

Fonte: Arquivo pessoal. 
Após 24 horas, o animal foi operado para correção do defeito da fenda palatina e exérese da massa ocular. O préoperatório, consistiu no uso de $2,5 \mathrm{mg} / \mathrm{kg}$ de enrofloxacino e $0,5 \mathrm{mg} / \mathrm{kg}$ de meloxicam, ambos aplicados por via intramuscular.

A medicação pré-anestésica utilizou $0,15 \mathrm{mg} / \mathrm{kg}$ de cloridrato de xilazina a $2 \%$ e $15 \mathrm{mg} / \mathrm{kg}$ de cloridrato de cetamina e $0,1 \mathrm{mg} / \mathrm{kg}$ de midazolam, associados na mesma seringa e aplicados pela via intramuscular com manutenção anestésica realizada pela infusão contínua de propofol na taxa de $0,5 \mathrm{mg} \mathrm{kg} / \mathrm{min}$. pela via intravenosa e mantido em respiração espontânea apenas com auxílio de máscara de oxigênio a um fluxo de $3 \mathrm{~L} / \mathrm{min}$.

O animal foi posicionado em decúbito lateral esquerdo, e realizada antissepsia com iodo-povidona a $0,02 \%$ seguida de exérese da massa, com elevação da terceira pálpebra e incisão em cunha. A síntese cirúrgica foi realizada com fio de poliglactina 910 (5-0) em sutura de padrão simples separado.

O pós-operatório consistiu na utilização de $0,5 \mathrm{mg} / \mathrm{kg}$ de meloxicam a $2 \%$, por via intramuscular, SID e 2,5 mg/kg de enrofloxacino por via intramuscular, SID, ambos administrados durante 3 dias consecutivos, e associados a limpeza com iodopovidona a $0,02 \%$ da cavidade bucal.

A massa proveniente da exérese foi encaminhada ao exame histopatológico, medindo $0,5 \times 0,3 \mathrm{~cm}$. Na histologia foi possível a observação de epitélio escamoso estratificado, folículos pilosos, glândulas sebáceas, tecido adiposo, tecido fibroso e feixes finos de fibras musculares lisas, compatível com dermoide (Figura 1B).

O pós-operatório tardio, contou com a observação do animal por 15 dias, onde segundo informações do proprietário, não foram identificadas recidivas ou lesões em terceira pálpebra direita.

\section{Resultados e Discussão}

Alterações congênitas são anomalias que podem se desenvolver em todas as espécies em diferentes regiões anatômicas, afetando parcial ou totalmente tecidos e órgãos, as quais se desenvolvem durante o período embrionário ou fetal (Rotta, Torres, \& Motta, 2008). Como agente etiológico temos infecções, intoxicações, lesões durante o período gestacional e deficiências nutricionais. Contudo a ocorrência de doenças congênitas também pode estar relacionada a fatores hereditários ou ocorrer de forma esporádica, sem estar associada necessariamente a algum fator específico (Campos et al., 2009; Dantas et al., 2010). Nossos achados corroboraram com estudos anteriores pois no histórico familiar do animal, nenhum dos progenitores ou animais do rebanho da propriedade, apresentavam manifestações de dermoide ocular.

A incidência de cistos dermoides na região ocular é relativamente baixa em bovinos, possuindo uma prevalência estimada de $0,002 \%$ a 0,4\%, com exceção para os animais da raça Hereford (Kiliç, Toplu, \& Epikmen, 2012). Essa baixa incidência pode acarretar em dificuldade na detecção de alterações por parte dos tratadores ou proprietários dos animais, por ocasião de sua manifestação, o que pode acarretar danos futuros como queda na produtividade e lesões de maior gravidade. Portanto, ressalta-se a importância da identificação, diagnóstico e terapia adequadas.

Meyer (1995), classificou o cisto dermoide como uma formação cística revestida por um epitélio escamoso semelhante a pele, corroborando com o evidenciado pela análise histológica. Além dos achados presentes compatíveis com dermoide, em alguns casos como relatado por Macnel e Moxham (2010) e por Gordon, Faquin, Lahey, e Kaban (2013) pode ocorrer a presença de glândulas sudoríparas, vasos sanguíneos, epitélio queratinizado, tecido adiposo, tecido nervoso e em outros o desenvolvimento de tecido cartilaginoso.

Além das estruturas presentes no dermoide, outro ponto a ser considerado é a forma de apresentação, variando entre uma forma achatada ou, como descrita por Castro, Hendrix, a Van Amstel (2006), de forma pendular de maneira individual ou em várias partes do mesmo olho, o que não foi observado nesse caso.

Lesões secundárias podem se desenvolver devido ao tamanho e localização do dermoide, e a presença de pelos no cisto, quando em contato direto com o olho do animal, pode comprometer o globo ocular, causando desde um incomodo até 
uma irritação grave com ulcerações na córnea, além de blefaroespasmo, epífora, secreções oculares e até mesmo cegueira (Brudenall et al., 2008; Cunha, 2008; Carniel \& Leseux, 2018). Entretanto, se o dermoide for diagnosticado precocemente menor serão as chances de desenvolver lesões graves ao globo ocular sendo necessária a enucleação. No presente caso, o diagnóstico e conduta cirúrgica ocorreram precocemente.

Outra complicação que deve ser ressaltada, é quanto ao local onde o dermoide se apresenta. As pálpebras exercem importante função para proteger os olhos assim como a terceira pálpebra, que tem como função a produção de lágrimas, proteção imunológica e proteção mecânica da superfície do olho (Ward, 1999). O dermoide, quando presente na pálpebra ou terceira pálpebra pode, impedir o movimento de oclusão da pálpebra, provocando infecções bacterianas, uma vez que a distribuição da película lacrimal passa a ser afetada (Almeida et al., 2018).

Quanto ao tempo de identificação do dermoide, a idade do animal é relevante, pois na maioria dos casos o processo cicatricial é mais rápido em indivíduos jovens, quando comparados a indivíduos adultos ou idosos (Biondo-Simões, Ioshii, Borsato, \& Zimmermann, 2005). Esse estudo corrobora com as afirmações dos autores, considerando que no pós-operatório, não ocorreram intercorrências.

O procedimento de eleição para o tratamento do cisto dermoide é a exérese cirúrgica que possui como finalidade além do tratamento, a obtenção de um diagnóstico histopatológico, prevenção de infecções e também finalidades estéticas (Pereira et al.,2000). Na maioria dos casos, o procedimento adotado é a ceratectomia lamelar superficial que consiste na remoção da camada superficial da córnea. Entretanto esse procedimento não é empregado em todos os casos de dermoide ocular, devido ao fato de que o cisto pode se apresentar em outras regiões como descrito, O caso relatado, obteve êxito cirúrgico e ausência de recidiva.

Existem alternativas que podem ser adotadas para o tratamento e remoção de cistos dermoide como a crioterapia, hipertermia e a radiofrequência (Seward, 1965; Sarrafzadeh-Rezaei et al., 2007). Contudo, optou-se pela exérese cirúrgica, em virtude de utilizar um único procedimento anestésico para realização de outra abordagem cirúrgica.

Em animais de rebanho, a identificação de enfermidades como o cisto dermoide na região ocular pode ser mais desafiadora, pois em muitos casos, os animais que apresentam estas enfermidades não são removidos do rebanho para realizar o tratamento e com isso as complicações podem se tornar significativas, a ponto de comprometer a qualidade de vida destes animais e c seu valor zootécnico (Brudenall et al., 2008; Almeida et al., 2018). A observação do cisto dermoide nesse caso só ocorreu após o animal ser encaminhado a atendimento médico veterinário pela queixa inicial de regurgitação porém nenhuma alteração ocular havia até então, sido observada pelo proprietário.

O pós-cirúrgico foi essencial para o sucesso do procedimento, posto que o processo cicatricial pode ser afetado por fatores externos como bactérias, fungos, sujidades entre outros, tendo como porta de entrada a incisão cirúrgica e em alguns casos comprometendo a visão do animal. Por tanto a utilização de medicamentos de uso tópico como antibióticos, antiinflamatórios foi fundamental neste período. No pós-operatório além da utilização de antibiótico e antiinflamatório de uso sistêmico, foi orientada apenas a limpeza com solução fisiológica, BID em olho direito (OD). O animal foi reavaliado após 10 dias, mostrando boa cicatrização no local operado.

\section{Conclusão}

O cisto dermoide mesmo sendo de baixa ocorrência nos rebanhos bovinos, não deve ser negligenciado, pois são grandes as complicações oftálmicas geradas pela sua permanência no globo ocular. Esse estudo enfatiza a importância do diagnóstico e conduta terapêutica precoces, para manutenção da qualidade de vida do animal e manutenção de ganhos zootécnicos aos animais de produção. 


\section{Referências}

Albuquerque, L., Freitas, L. V. R. P., Hünning, P. S., Bercht, B. S., \& Pigatto, J. A. T. (2012). Dermóide ocular - revisão de literatura. Medvep - Revista Científica de Medicina Veterinária, 10(32), 44-47.

Almeida, F. F., Seal, D. C. M., Mayer, L. L., Fernandes, N. S., Ferreira, H. I. P., \& Junior, R. A. B. (2018). Soro autólogo no controle bacteriano, em bovino submetido a excisão cirúrgica de dermóide ocular. Ciência Animal, 28(3), 99-105.

Barkyoumb, S. D., \& Leipold, H. W. (1984). Nature and cause of bilateral ocular dermoids in Hereford cattle. Veterinary Pathology, $21(3), 316-324$.

Biondo-Simões, M. D. L. P., Ioshii, S. O., Borsato, K. S., \& Zimmermann, E. (2005). O processo de cicatrização influenciado pelo hipotireoidismo e pelo envelhecimento: estudo da cicatrização da parede abdominal, em ratos. Acta Cirúrgica Brasileira, 20, 120-128.

Brudenall, D. K., Ward, D. A., Kerr, L. A., \& Newman, S. J. (2008). Bilateral corneoconjunctival dermoids and nasal choristomas in a calf. Veterinary ophthalmology, 11(3), 202-206.

Campos, K. F., Sousa, M. G. S., Silva, N., Oliveira, H. S., Duarte, M. D., Barbosa, J. D., \& Oliveira, C. M. C. (2009). Doenças congênitas em bovinos diagnosticadas pela Central de Diagnóstico Veterinário (CEDIVET) da Universidade Federal do Pará, no período de 1999 a 2009. Ciência Animal Brasileira, $13-18$.

Canelas, H. M., Cruz, O. R., \& Tenuto, R. A. (1960). Tumores congênitos do sistema nervoso I-cistos epidermóides e dermóides. Arquivos de NeuroPsiquiatria, 18(3), 209-223.

Carniel, T. O. L., \& Leseux, C. (2018). Relato de caso: tratamento com plasma rico em plaquetas (PRP) pós-ressecção de dermoide límbico em cão. In: Anais do Congresso Nacional de Medicina Veterinária FAG, 2(1).

Castro, J. R., Hendrix, D. V., \& Van Amstel, S. R. (2006). Ocular Dermoids in Cattle: A Case Report and Review. The Bovine Practitioner, 40(1), 8-12.

Cunha, O. (2008). Manual de oftalmologia veterinária. Afecções da córnea - Dermóide, Brasil: Universidade Federal Do Paraná.

Dantas, A. F. M., Riet-Correa, F., Medeiros, R. M., Galiza, G. J. N. D., Pimentel, L. D. A., Anjos, B. L. D., \& Mota, R. A. (2010). Congenital malformations in ruminants in the semiarid of the Brazilian Northeast. Pesquisa Veterinária Brasileira,30(10), 807-815.

Gordon, P. E., Faquin, W. C., Lahey, E., \& Kaban, L. B. (2013). Floor-of-mouth dermoid cysts: report of 3 variants and a suggested change in terminology. Journal of oral and Maxillofacial Surgery, 71(6), 1034-1041.

Kiliç, N., Toplu, N., \& Epikmen, E. T. (2012). Surgical treatment of corneal large dermoid in a simmental calf. Acta Scientiae Veterinariae, $40(2), 1-5$.

Leipold, H. W. (1984). Congenital ocular defects in food-producing animals. The Veterinary clinics of North America. Large animal practice, 6(3), 577 - 595.

Macneil, S. D., \& Moxham, J. P. (2010). Review of floor of mouth dysontogenic cysts. Annals of Otology, Rhinology \& Laryngology, $119(3), 165-173$.

Meyer, I. (1955). Dermoid cysts (dermoids) of the floor of the mouth. Oral Surgery, Oral Medicine, Oral Pathology, 8(11), 1149-1164.

Muñoz, E., Leiva, M., Naranjo, C., \& Pena, T. (2007). Retrobulbar dermoid cyst in a horse: a case report. Veterinary Ophthalmology, $10(6), 394-397$.

Parrah, J.D., Moulvi, B.A., Athar, H., Mir, M.S., U Din, M., Gazi, M., \& Handoo, N. A. (2013). Retrospective study on the surgical affections of young calves. Journal of Advanced Veterinary Research, 3(1), 77-82.

Pereira, C. U., Santos, D. P. D. S., Machado, J. D. C., Machado, S. D. C., Araújo, E. S. D., \& Costa, M. D. (2000). Cisto dermoide de inclusão congênita localizada na fontanela anterior: relato de dois casos. Arquivos Brasileiros de Neurocirurgia, 19(1), 32-35.

Roh, Y. S., Gi, D. B., Lim, C. W., \& Kim, B. (2014). Asymmetrical ocular dermoid in native Korean cattle. The Journal of animal \& Plant Sciences, 24(3), 976-978.

Rotta, I. T., Torres, M. B. A. M., \& Motta, R. G. (2008). Diprosopia em bovino. Arquivo Brasileiro de Medicina Veterinária e Zootecnia, 60(2), 489-491.

Sarrafzadeh-Rezaei, F., Farshid, A. A., \& Saifzadeh, S. (2007). Congenital ocular dermoid cyst in a river buffalo (Bubalus bubalis) calf. Journal of Veterinary Medicine Series A, 54(1), 51-54.

Seward, G. R. (1965). Dermoid cysts of the floor of the mouth. British Journal of Oral Surgery, 3, 36-47.

Souza, F. V. D., Nogueira, R. L. M., Turatti, E., Menezes Júnior, J. M. S., \& Freitas, M. R. (2008). Cisto dermoide: relato de caso clínico. Revista de cirurgia traumatol. buco-maxilo-facial, 7(4), 37-42.

Souza, N. F., Pereira, W. L. A., Andrade, S. L. S., Moura, M. A. O., Mileo, V. C., Lima, M. M., \& Jaques, A. M. D. C. C. (2020). Ovários policisticos epidermóides e hipoplasia uterina em uma vaca bovina. Acta Scientiae Veterinariae, 48(1), 488-493.

Steinmetz, A., Locher, L., Delling, U., Ionita, J. C., Ludewig, E., Oechtering, G., \& Wittek, T. (2009). Surgical removal of a dermoid cyst from the bony part of the nasolacrimal duct in a Scottish highland cattle heifer. Veterinary Ophthalmology, 12(4), 259-262.

Stelmann, U. J. P., Souza, B. G. D., Araujo, P. C. M., Monteiro, G. A., Seppa, G. S., \& Silva, A. A. (2009). Cisto dermoide em equino: relato de caso. Revista Científica Eletrônica de Medicina Veterinária, 7(13),1-9.

Utumi, E. R., Zambon, C. E., Pedron, I. G., Machado, G. G., \& Rocha, A. C. (2010). Recidiva de cisto dermoide congênito de localização paramediana. Arquivos Internacionais de Otorrinolaringologia, 14(3), 368-372. 
Research, Society and Development, v. 10, n. 5, e5010514586, 2021

(CC BY 4.0) | ISSN 2525-3409 | DOI: http://dx.doi.org/10.33448/rsd-v10i5.14586

Wagner, F., Brügmann, M., Drommer, W., \& Fehr, M. (2000). Corneal dermoid in a dwarf rabbit (Oryctolagus cuniculi). Journal of the American Association for Laboratory Animal Science, 39(5), 39-40.

Ward, D. A. (1999). Diseases and surgery of the canine nictitating membrane. Veterinary ophthalmology, 3, 609-618. 